\title{
Keratinocyte Conjugation by Adhesion Peptide Induces Up-Regulation of Tight Junction Proteins and Immune Regulation Under Inflammatory Conditions
}

\author{
Jun-Ho Lee ${ }^{1,2 \#}$, So-Yeon Choi ${ }^{1 \#}$, Soo-Yeoun Park ${ }^{1}$, Nam-Chul Jung ${ }^{2}$, Ji-Soo Oh' ${ }^{1}$, Jinjung Choi ${ }^{3}$, Ji \\ Hyung Chung ${ }^{1}$, Jie-Young Song ${ }^{4}$, Han Geuk Seo ${ }^{5}$ and Dae-Seog Lim ${ }^{1 *}$ \\ ${ }^{1}$ Department of Biotechnology, CHA University, Seongnam-si, Gyeonggi-do, Republic of Korea
}

${ }^{2}$ Pharos Vaccine Incorporated, Seongnam-si, Gyeonggi-do, Republic of Korea

${ }^{3}$ Department of Internal Medicine, Division of Rheumatology, Bundang CHA Medical Center, Seongnam-si, Gyeounggi-do, Republic of Korea

${ }^{4}$ Department of Radiation Cancer Sciences, Korea Institute of Radiological and Medical Sciences, Seoul, Republic of Korea

${ }^{5}$ Department of Food Science and Biotechnology of Animal Products, Sanghuh College of Life Sciences, Konkuk University, Seoul, Republic of Korea

\#These authors contributed equally to this study

*Corresponding author: Dae-Seog Lim, Department of Biotechnology, CHA University, Seongnam-si, Gyeonggi-do, Republic of Korea

\section{ARTICLE INFO}

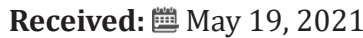

Published: 慧 June 02, 2021

Citation: Jun-Ho Lee, So Yeon Choi, Soo Yeoun Park, Nam Chul Jung, Dae-Seog Lim, et al., Keratinocyte Conjugation by Adhesion Peptide Induces Up-Regulation of Tight Junction Proteins and Immune Regulation Under Inflammatory Conditions. Biomed J Sci \& Tech Res 36(2)-2021. BJSTR. MS.ID.005820.

Abbreviations: TJs: Tight Junctions; APCs: Antigen Presenting Cells; DCs: Dendritic Cells; imDC: Immature DCs

\section{ABSTRACT}

Keratinocyte Tight Junctions (TJs) in the epidermal layer play a crucial role in skin barrier function. An effective immune response in skin is usually initiated by dendritic Antigen Presenting Cells (APCs) in the epidermis and dermis and is finally executed by T lymphocytes. The aim of this study was to investigate whether increased keratinocyte TJ proteins affect immune responses mediated by Immature Dendritic Cells (imDCs). Keratinocytes were treated with the peptide AdhPep-10-2 (10 nM) and then cocultured with DCs for $24 \mathrm{~h}$. Cytokine production was measured by ELISA and a Luminex Multiplexing System. DCs and T cell subsets were analyzed by flow cytometry. Expression of TJ proteins by keratinocytes treated with AdhPep-10-2 increased markedly, whereas CXCL1 chemokine secretion decreased. Coculture of imDCs with AdhPep-10-2-treated keratinocytes inhibited maturation of DCs, reduced production of pro-inflammatory cytokines, and increased the regulatory $\mathrm{T}$ (Treg) cell population in the presence of LPS. These results suggest that AdhPep-10-2 peptide increases keratinocyte TJ proteins, thereby inducing anti-inflammatory responses by imDCs under inflammatory conditions. Taken together, these studies show strong possibility that increased keratinocyte TJ proteins play a key role in DC-mediated immunity.

\section{Introduction}

The skin, the body's largest organ, helps to secure the integrity of the host and allows the individual to communicate with the outside world. A network of physical, chemical, and immunologic components ensures a finely tuned balance between protection from harmful pathogens and bidirectional signal exchange [1,2]. Immune responses originating in the skin are executed by cells and molecules belonging to the innate and adaptive immune systems. The innate immune system, which provides immediate host defense, includes physical barriers (e.g., epithelia) and soluble factors such as antimicrobial peptides, chemokines, and cytokines produced by a variety of resident cells (e.g., keratinocytes and fibroblasts) and infiltrating leukocytes (e.g., granulocytes, monocytes/macrophages, and Dendritic Cells (DCs)) [1-3]. The most numerous cell within the epithelial compartment is the keratinocyte [1,3]. In addition to its important role in maintaining the physical barrier of the skin, the keratinocyte actively participates in innate immune responses. When keratinocytes are stimulated by danger signals (e.g., LPS), they release large amounts of cytokines that trigger inflammation, 
activate/inhibit $\mathrm{T}$ cells, modulate the phenotype and function of Langerhans cells (skin DCs), and regulate leukocyte trafficking [13]. An effective immune response in human skin is usually initiated by dendritic Antigen-Presenting Cells (APCs) and is ultimately executed by $\mathrm{T}$ lymphocytes or B cell-derived antibodies, or both $[1,2]$.

DCs are professional APCs that play major roles in initiating and regulating adaptive immune responses. They are capable of capturing and processing antigens, migrating into lymphoid organs, stimulating antigen-specific responses by naïve resting $\mathrm{T}$ cells, and secreting cytokines to initiate immune responses [2,46]. Immature DCs (imDC) regulate skin homeostasis by promoting the development of regulatory $\mathrm{T}$ cells, while their mature counterparts (i.e., DCs that have been stimulated by danger signals) induce cyto-destructive reactions [1,4-6]. An important type of immunomodulatory $\mathrm{T}$ cell that controls immune responses is the Treg cell, formerly known as the T suppressor cell [7]. Treg cells are induced by immature APCs/DCs and are the key to maintaining tolerance to self-antigens in the periphery [4-6]. Tight junction (TJ) proteins expressed by keratinocytes in the epidermal layer play a central role in barrier function. TJ proteins create a barrier by forming cell-cell junctions that connect cells together very tightly, which affects the function of the immune system in epidermal tissues [8].

The AdhPep-10-2 peptide (A-K-P-T-Y-K-A-K-P-T-Y-K-A-K-PT-Y-K) (INCI name: s-Mussel Oligopeptide-2; provided by Dr. Ji Hyung Chung, CHA University), a synthetic 18 amino acid peptide comprising three repeated sequences, resides within the 241-246 amino acid sequence of foot protein-1 of mytilus edulis; this protein promotes cell-cell interactions in keratinocytes in just 10 min [912]. This data suggested an effect of Adh-Pep-10-2 on tight junction formation [9-12]. In addition, AdhPep-10-2 increases expression of TJ proteins. To the best of our knowledge, no study has examined the relationship between increased TJ proteins and DC-mediated immunity; this was the aim of the present study.

\section{Materials and Methods}

\section{Ethics Statement}

This study was carried out in strict accordance with the recommendations in the Guide for the Care and Use of Laboratory Animals of the National Institutes of Health. The protocol was approved by the Institutional Animal Care and Use Committee of CHA University (The approved protocol Number: 180136). All surgery was performed under $\mathrm{CO}_{2}$ gas euthanasia, and all efforts were made to minimize suffering.

\section{Mice}

Male Balb/c mice (6-8 wk of age, 14-16 g, $\mathrm{n}=30$ ) were purchased from Orient Bio Inc. (Gyeonggi, Korea) and housed in a temperature- and humidity-controlled room under a $12 \mathrm{hr} / 12$ hr light/dark cycle. These animals were obtained for use in other ongoing studies.

\section{Cell Culture}

MCA-3D keratinocyte cells (originated in Balb/c mice) were supplied by CLS Cell Lines Service GmbH (Germany) and cultured in Ham's F10 (Lonza, Walkersville, MD, USA) supplemented with $10 \%$ fetal bovine serum (FBS, certified US origin; Gibco, Life Technologies, Grand Island, NY, USA) and 100x AntibioticsAntimycotics (A.A) (Gibco). Keratinocytes were treated (or not) with AdhPep-10-2 (10 nM) (provided by Dr. Chung, CHA University) for $18 \mathrm{~h}$. DCs were generated from bone marrow progenitor cells isolated from Balb/c mice [4-6]. Briefly, bone marrow cells were cultured in RPMI 1640 supplemented with HEPES (Lonza), 10\% FBS (Gibco), 100x A.A (Gibco), $20 \mathrm{ng} / \mathrm{mL}$ recombinant mouse (rm) GM-CSF (JW CreaGene, Gyeonggi, Korea), and 2 ng/mL rmIL-4 (JW CreaGene). Cells were maintained at $37^{\circ} \mathrm{C}$ under an atmosphere containing $5 \% \mathrm{CO}_{2}$. On Day 3, fresh medium containing the same concentrations of GM-CSF and IL-4 was added. On Day 6, half of the culture medium was replaced with fresh medium. After 8 days of culture, DCs were harvested and mature dendritic cells generated by additional incubation for $24 \mathrm{~h}$ with $1 \mu \mathrm{g} / \mathrm{mL}$ LPS (SigmaAldrich) (; DC+LPS). DCs and DCs+LPS were harvested at the same time for use in experiments. Splenocytes were isolated from Balb/c mice and disaggregated in RPMI 1640 medium. CD3 ${ }^{+}$T cells were enriched by passing the splenocytes through a nylon wool (Polysciences Inc., Warrington, PA, USA) column. Splenocytes were cultured in AdhPep-10-2 (or not) and LPS-treated keratinocyte culture supernatant (PKS or KS; concentrated 20-fold) diluted 1:1 in RPMI 1640 medium supplemented with 10\% FBS, 100x A.A, and $100 \mathrm{IU} / \mathrm{mL}$ IL-2.

\section{Co-Cultures}

Keratinocyte (adherent; $5 \times 10^{4}$ cells $/ \mathrm{mL}$ ) were treated (or not) with $10 \mathrm{nM}$ AdhPep-10-2 for $18 \mathrm{~h}$. After washing out, DCs or DCs+LPS (suspension; 1 x $10^{5}$ cells $/ \mathrm{mL}$ ) were co-cultured for 24 h (; Pep-kera-DCs or Kera-DCs or Pep-kera-DCs+LPS or KeraDCs+LPS). Pep-KeraDCs+LPS or Kera-DCs+LPS $\left(1 \times 10^{5}\right.$ cells $\left./ \mathrm{mL}\right)$ separated from the keratinocytes were then co-cultured with $\mathrm{T}$ cells $\left(1 \times 10^{6}\right.$ cells $\left./ \mathrm{mL}\right)$ for $72 \mathrm{~h}$ at $37^{\circ} \mathrm{C} / 5 \% \mathrm{CO}_{2}$ in $2 \mathrm{~mL} \mathrm{RPMI} 1640$ supplemented with $10 \%$ FBS and 100x A.A.

\section{Confocal Microscopy}

Keratinocytes stained with PKH67 (green) or CellVue Burgundy (red) (according to the manufacturer's protocol) fluorescent dyes (ThermoFisher Scientific) were co-cultured for $1 \mathrm{~h}$. PKH67 and CellVue Burgundy were used proprietary membrane labeling technology. Briefly, PKH67-labeled keratinocytes were precultured for 20 min with Adh-10-2 peptide at $37^{\circ} \mathrm{C} / 5 \% \mathrm{CO}_{2}$ shaking incubator in conical tube. After washing with PBS, PKH67-labelled keratinocytes were co-cultured for $1 \mathrm{~h}$ with CellVue Burgundy- 
labeled keratinocytes at $37^{\circ} \mathrm{C} / 5 \% \mathrm{CO}_{2}$ shaking incubator in conical tube. After washing, physical cell interaction was determined immediately in suspension by confocal microscopy. Images were imported into the free image processing software ImageJ. ImageJ includes multiple pre-installed plugins. We subtracted the background of both channels after thresholding of signal-containing areas with the 'Older colocalization plugins; Colocalization Threshold'. Next, we used 'ImageJ plugins for colocalization analysis; Colocalization Finder'. The Colocalization Finder plugin displays a correlation diagram from two initial pictures having the same size together with a RGB overlap of the original images. Drawing a rectangular selection on this diagram allows you to highlight corresponding pixels on a RGB overlap of the original images, and if selected, on a $3^{\text {rd }}$ image. Analysis can be restricted to pixels having values with a minimum ratio. Selection settings are logged to a results window. The results window was displayed the calculated Pearson's correlation, \% colocalization, and some other details of the test calculation (; contribution of both channels to overlap coefficient, slope and intercept of the linear regression, \% pixels selected, min and max in both channels).

\section{Western Blot Analysis}

Keratinocytes treated (or not) with AdhPep-10-2 were lysed with pro-prep extraction buffer (iNtRON, Gyeonggi, Korea) containing a 5-protease inhibitor cocktail. The protein concentration was then measured using a BCA protein assay kit (Pierce, Rockford, IL, USA). Equal amounts of protein were separated on SDS-PAGE gels and transferred to PVDF membranes (BioRad, CA, USA). The membranes were blocked with 5\% skim milk in PBST and then incubated overnight at $4^{\circ} \mathrm{C}$ with primary antibodies as follows: anti-occludin rabbit mAb (E6B4R, Cell Signaling Technologies, Danvers, MA, USA), anti-claudin-1 rabbit mAb (D3H7C, Cell Signaling Technologies), or anti-GAPDH rabbit mAb (14C10, Cell Signaling Technologies); all diluted 1:1000. The membranes were then washed with PBST and incubated for $2 \mathrm{~h}$ at room temperature with an HRP-conjugated secondary antibody (7074, Cell Signaling Technologies); diluted 1:2000. The membrane was then exposed to ECL reagents (Thermo Scientific) and signals were detected using a Luminescent image analyzer (LAS-4000, Fuji Film, Tokyo, Japan).

\section{Cytokine Measurement}

The amounts of interleukin-1 $\beta$ (IL-1 $\beta$ ), IL-6, IL-10, IL-17A, IL22 (BioLegend, CA, USA), IL-12p70, IL-4, interferon (IFN)- $\gamma$ (BD Biosciences), and CXCL1 (R\&D systems) in cell culture supernatants from lymphocytes or DCs alone were measured using commercial ELISA kits, according to the manufacturer's instructions. All samples were assayed in duplicate. A Luminex Multiplexing assay were performed using the Beadlyte Mouse 21-Plex Cytokine Detection System (Upstate Biotechnology, Lake Placid, NY, USA) according to the manufacturer's instructions. Keratinocyte culture supernatants were harvested and then incubated for $2 \mathrm{~h}$ with Beadlyte Mouse 21-
Plex Multi-Cytokine beads in the individual wells of a 96-well plate. Each sample was plated in duplicate. Next, $25 \mu \mathrm{l}$ of Beadlyte Mouse 21-Plex Multi-Cytokine biotin was added to each well and the plate was incubated for a further $1.5 \mathrm{~h}$. Diluted Beadlyte StreptavidinPhycoerythrin was added to each well ( $25 \mu$ l per well), incubated for $30 \mathrm{~min}$, and the plate was read on a Luminex xMAP 100 analyzer (Upstate Biotechnology). The cytokine concentrations were extrapolated from standard curves using BeadView Data Analysis software (Upstate Biotechnology). Data were analyzed using the MasterPlex QT2010 (MiraiBio, Hitachi, CA, USA).

\section{qRT-PCR}

RNA was isolated from keratinocytes treated (or not) with AdhPep-10-2 using TriZol reagent (Life Technologies, Mount Waverley, Australia). Total RNA was reverse transcribed using a SensiFAST ${ }^{\mathrm{TM}}$ cDNA Synthesis Kit (Bioline, London, UK) and cDNA subjected to real-time PCR analyses with specific primers and SensiFAST ${ }^{\text {TM }}$ SYBR. The primers used for real-time PCR were as follows: CXCL1, forward 5-GCT GGG ATT CAC CTC AAG AA-3 and reverse 5-TGG CTA TGA CTT CGG TTT GG-3; GAPDH, forward 5-AAC AGC AAC TCC CAC TCT TC-3 and reverse 5-CCT GTT GCT GTA GCC GTA TT-3. Expression of CXCL1 mRNA was normalized to that of $G A P D H$. All samples were run in duplicate.

\section{Flow Cytometry Analysis}

Fluorescently-conjugated Monoclonal Antibodies (mAbs) were used to examine the phenotype of DCs and lymphocytes. Briefly, cells were incubated on ice with appropriate antibodies for $20 \mathrm{~min}$ before washing with PBS and examination in a FACSCalibur flow cytometer (BD, Mountain View, CA, USA). For surface phenotype analysis, cells were stained with the following monoclonal markers: anti-CD11c (Clone; N418), anti-CD80 (Clone; 16-10A1), anti-CD86 (Clone; P03), anti-CD40 (Clone; 3/23), anti-CD54 (Clone; 3E2), anti-CD14 (Clone; Sa14-2), anti-CD4 (Clone; RM4-5), anti-CD8 $\alpha$ (Clone; 53-6.7), anti-CD25 (Clone; CP61), All antibodies were purchased from BD Biosciences. Before analysis of intracellular staining, cells were cultured in the presence of Monensin (1000x, sigma) $4 \mathrm{~h}$, and then cells were fixed/permeabilized using an intracellular staining kit (BD Bioscience) or a Foxp3 staining buffer set (eBioscience) according to the manufacturer's protocol. For analysis of intracellular staining, cells were stained with the following monoclonal markers: PE-conjugated IFN-p (XMG1.2), -IL-17A (TC11-18H10), and -Foxp3 (FJK-16 s) antibodies (all from BD Bioscience). After staining, cells were washed with PBS and examined in a FACSCalibur flow cytometer (BD). Data were analyzed using FlowJo software (TreeStar, Inc., San Carlos, CA, USA).

\section{Statistical Analysis}

Data are expressed as the mean \pm SD. Statistical comparisons were performed using one way analysis of $t$ tests or variance (ANOVA) followed by the Newman-Keul's test. Differences were 
considered statistically significant at $\mathrm{P}<0.05$. Statistical analysis was performed using GraphPad software (GraphPad Prism v5.0; GraphPad Software).

\section{Results}

\section{Function of AdhPep-10-2}

To confirm the function of AdhPep-10-2 for tight junction protein, we cultured Adh-10-2 peptide with keratinocytes stained with PKH or Cell Vue Burgundy cell labelling reagent for $1 \mathrm{~h}$. Confocal images revealed that AdhPep-10-2 increased the physical interaction between keratinocytes (Figures 1A \& 1B). AdhPep-10-2 significantly increased the Pearson's correlation coefficient (Rr) and percent of co-localization (Figure 1C). To examine the correlation between increased cell-cell interaction and expression of TJ proteins, we examined expression of TJ proteins by western blotting with antibodies specific for occludin and claudin-1 (Figure 1D). Cells were exposed to peptide (10 nM) for $18 \mathrm{~h}$. The results showed a marked increase in TJ proteins (occludin and claudin-1) expression by keratinocytes treated with $10 \mathrm{nM}$ of peptide (Figure 1).

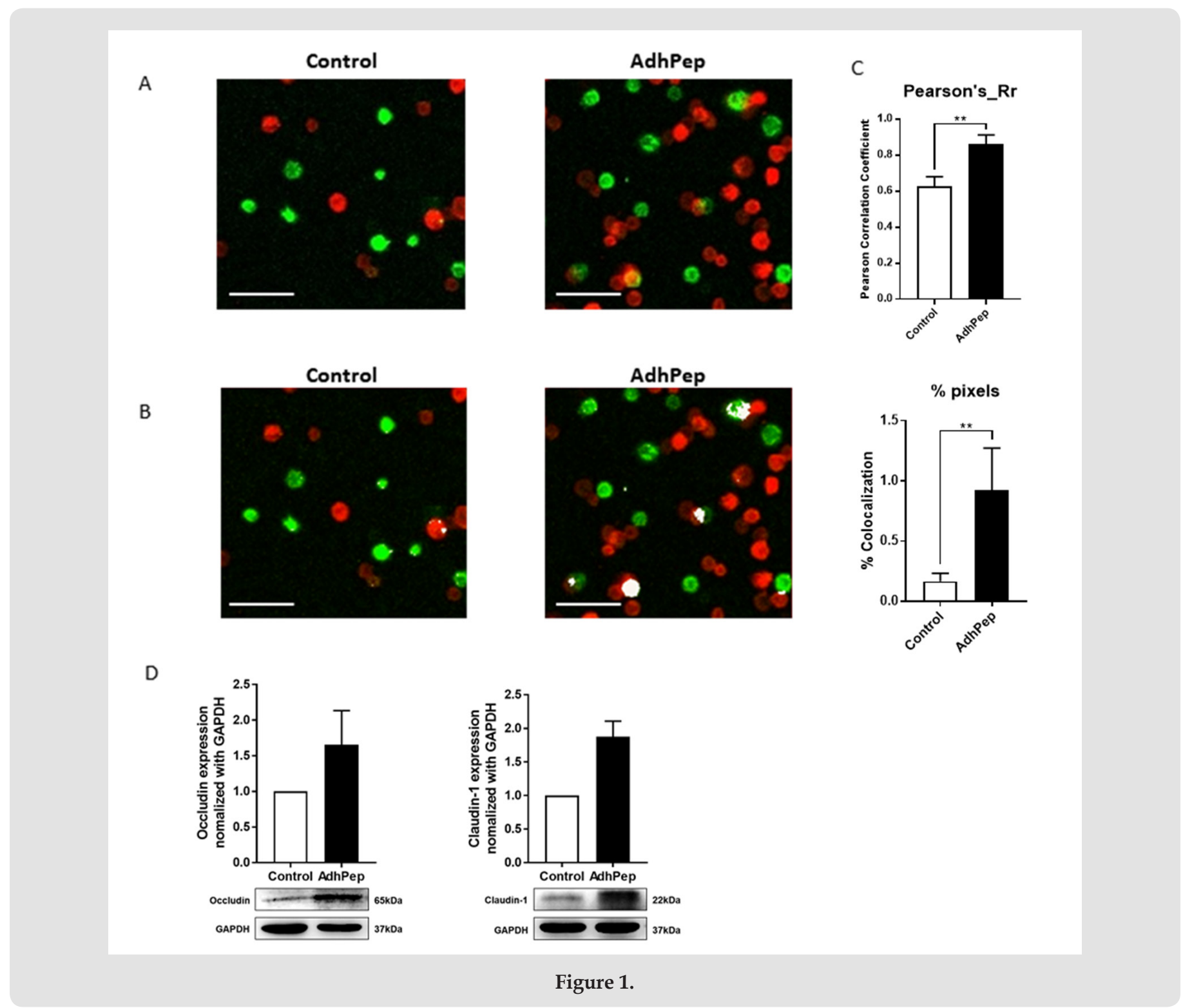

AdhPep-10-2 Increases Keratinocyte Tight Junction Proteins:

Representative images showing interaction between keratinocytes cultures with/without AdhPep-10-2. PKH-labeled keratinocytes (green) were co-cultured with CellVue Burgundylabeled keratinocytes (red) for $1 \mathrm{~h}$. After washing, physical interactions were observed under a confocal microscope. AdhPep-10-2 increased the physical interaction between keratinocytes. Scale bar: $100 \mu \mathrm{m}$.

a) The interactions are shown in yellow.

b) The interactions are highlighted in white. 
c) The Pearson's correlation coefficient (Rr) (upper) and percent of pixels (below) for co-localization was determined using Image J software. Data are expressed as mean \pm SEM ( $n=4$ individual enlarged pictures).

d) Expression of tight junction proteins including and claudin-1 by keratinocytes stimulated (or not) with AdhPep-10-2 (10 $\mathrm{nM}$ ) for $18 \mathrm{~h}$ and then lysed. The amount of tight junction proteins in the cell lysates was determined by western blotting with specific antibodies. All trials were performed in triplicate (mean $\pm \mathrm{SEM}$ ). ${ }^{* *} P<0.01$.

\section{Characterization of DCs Co-Cultured with AdhPep-10-2- treated Keratinocytes}

DCs generated from bone marrow progenitor cells showed high expression of CD11c, costimulatory molecules CD80, CD86, and CD40, and the cell adhesion molecule CD54. DCs expressed significantly lower levels of CD40, CD54, CD80, and CD86 (associated with DC maturation) than DC stimulated with LPS (DC+LPS, mature DCs; Figure 2A). Likewise, DCs produced lower levels of pro-inflammatory cytokines (IL-1 $\beta$, IL-6, and IL-12p70) than DC+LPS (Figure 2B). To examine the effect of AdhPep-10-2-treated keratinocytes on DCs, we are co-cultured both cell types. There was no significant difference in expression of markers associated with DC maturation or in production of pro-inflammatory cytokines between DCs co-cultured with AdhPep10-2-treated keratinocytes (Pep-Kera-DCs) and DCs co-cultured with untreated keratinocytes (no AdhPep-10-2; Kera-DCs) in the absence of LPS. However, PepKera-DCs stimulated with LPS (Pep-Kera-DC+LPS) showed lower expression of CD40, CD80, and CD86 (Figure 2C) than Kara-DCs stimulated with LPS (Kera-DC+LPS). A full gating strategy is shown in Figure S1. Moreover, in LPS condition, Pep-Kera-DCs produced significantly less IL-6 and IL-12p70, but not IL-1 $\beta$ (Figure 2D).

(A)

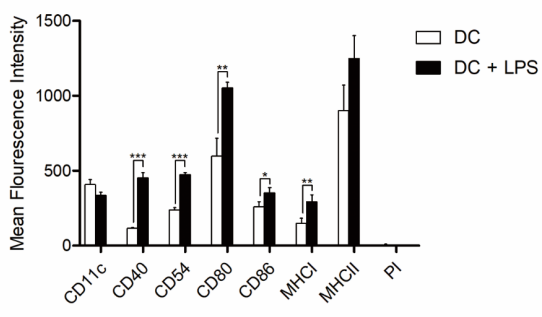

(B)

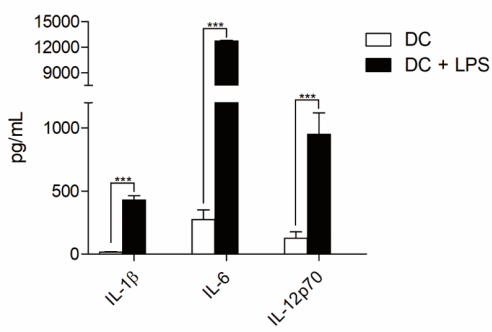

(C)
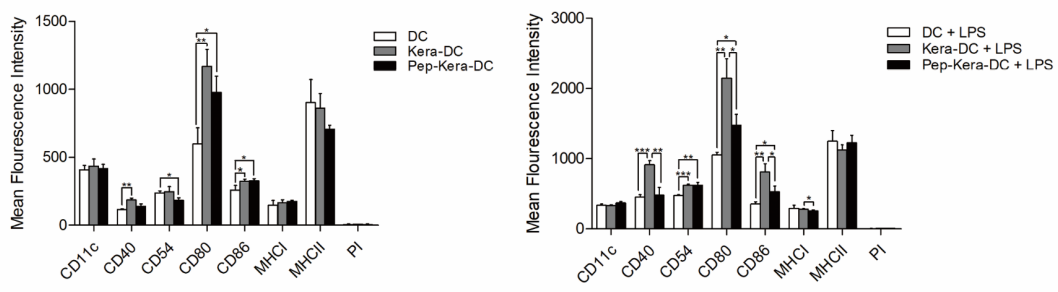

(D)
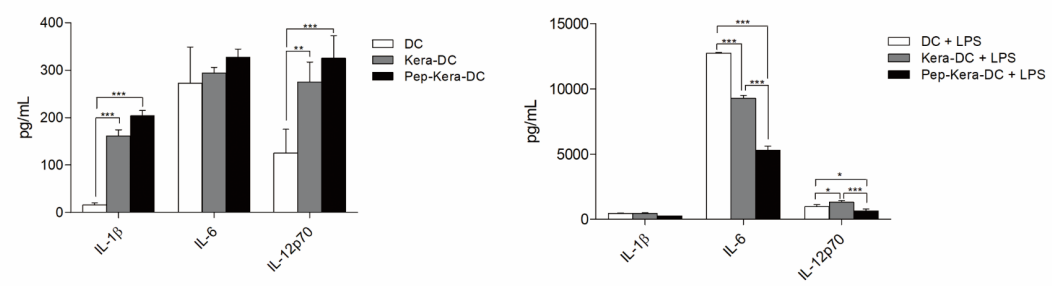

Figure 2. 


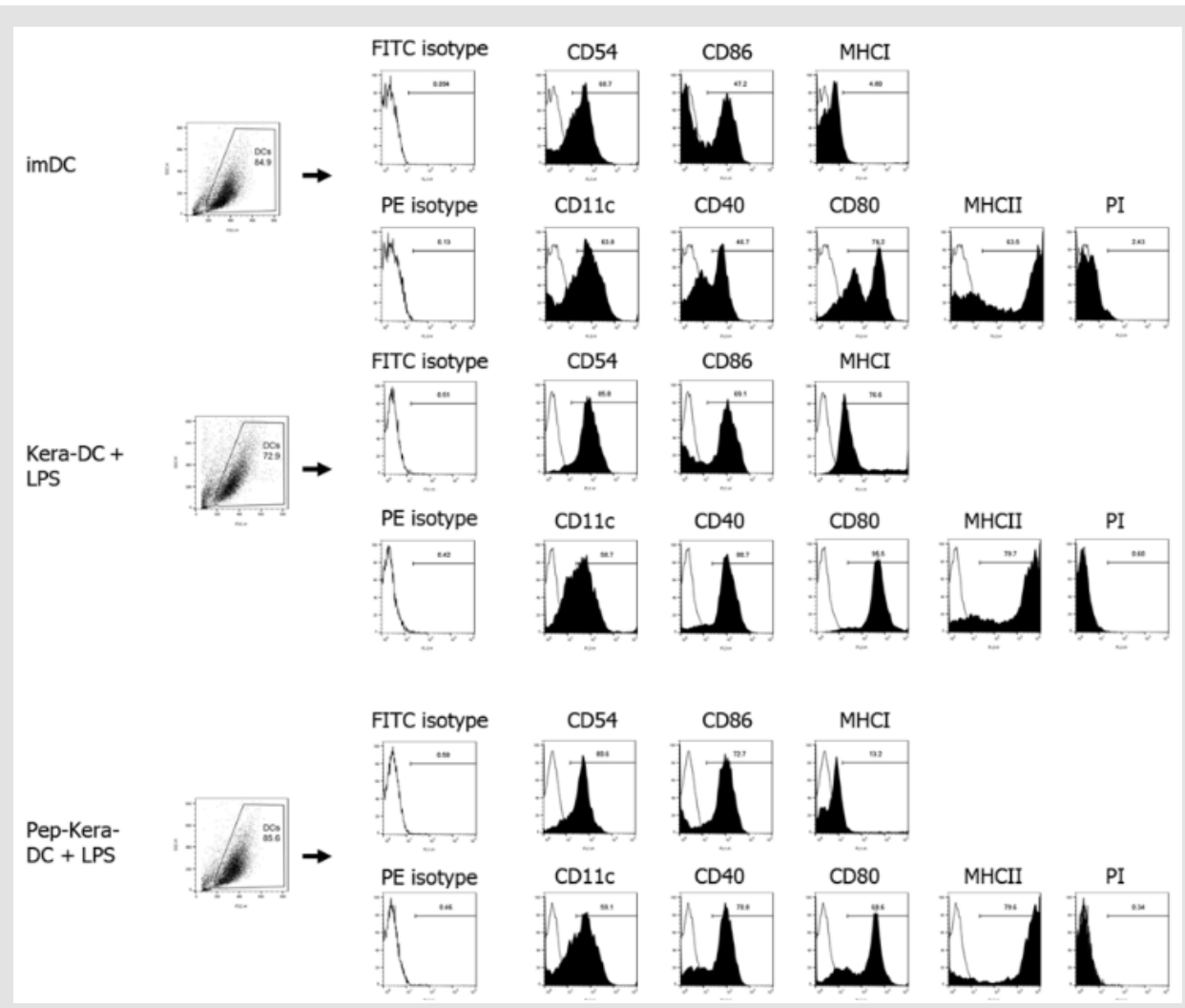

Figure 1S: Flow cytometry gating strategy for dendritic cells surface phenotype analysis. Initially, a broad scatter gate that excludes debris and the majority of dead cells was applied. Dead cells were excluded by uptake of Propidium Iodide (PI).

\section{Characterization of AdhPep-10-2-exposed DCs:}

a, c) DC subsets (DC, DC+LPS, Pep-kera-DCs, Kera-DCs, PepKera-DCs+LPS, and Kera-DCs+LPS) were stained with the indicated fluorescently-conjugated antibodies and analyzed by flow cytometry. Data are presented as bar graphs showing mean fluorescence intensity (mean \pm SEM; $n=3$ independent DC preparations).

b, d) Pro-inflammatory cytokines in the culture supernatants of DC subsets (DC, DC+LPS, Pep-kera-DCs, Kera-DCs, Pep-KeraDCs+LPS, and Kera-DCs+LPS) were measured by ELISA. Data are expressed as the mean \pm SEM $(n=3$ independent DC preparations) of duplicate experiments. ${ }^{*} P<0.05$; ${ }^{* *} P<0.01$; $* * * P<0.001$.

\section{Pep-Kera-DCs induce T cell Anti-Inflammatory Respons- es upon Co-Culture}

Next, we performed co-culture experiments to investigate the effects of Pep-Kera-DCs on Treg cell expansion and polarization of $\mathrm{CD}^{+} \mathrm{T}$ cells in the presence of LPS. Compared with Kera-DCs, PepKera-DCs induced a marked increase in the $\mathrm{CD} 4^{+} \mathrm{CD} 25^{+} \mathrm{FoxP} 3^{+}$Treg cell population (Figure 3A, a full gating strategy is shown in Figure S2) and inhibited production of IFN- $\gamma$ (Th1), IL-17A, and IL-22 (Th17). However, no Th2 cytokines (IL-4 and IL-10) were detected (Figure 3B). These results suggest that Pep-Kera-DCs induce antiinflammatory responses in $\mathrm{CD}^{+} \mathrm{T}$ cells upon co-culture by inducing generation of Treg cells.

Immunosuppressive Characteristics of DCs Co-Cultured with T cells:

a) Each DC subset (pep-kera-DC+LPS, kera-DC+LPS) was cocultured for $72 \mathrm{~h}$ with $\mathrm{T}$ cells. The stimulator responder ratio was 1:10. To identify Tregs, cells were stained with anti-CD4, anti-CD25 and anti-Foxp3 antibodies in Foxp3 staining buffer set and then analyzed by flow cytometry. The bar graph shows the percentage of $\mathrm{CD} 4^{+} \mathrm{CD} 25^{+} \mathrm{Foxp} 3^{+}$cells (mean $\pm \mathrm{SEM}$ of six independent co-culture preparations).

b) Cytokine levels in the supernatants after $72 \mathrm{~h}$ of co-culture, as measured by ELISA. Data are expressed as mean \pm SEM $(n=3$ independent co-culture preparations performed in duplicate). $* \mathrm{P}<0.05 ; * * \mathrm{P}<0.01$. 
(A)

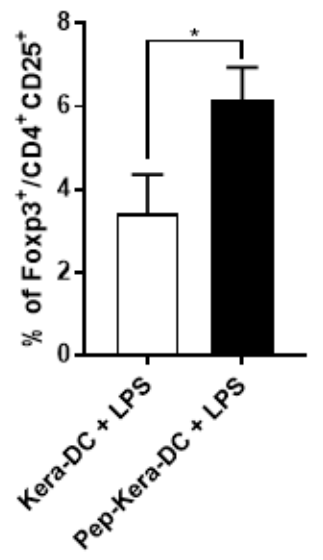

(B)

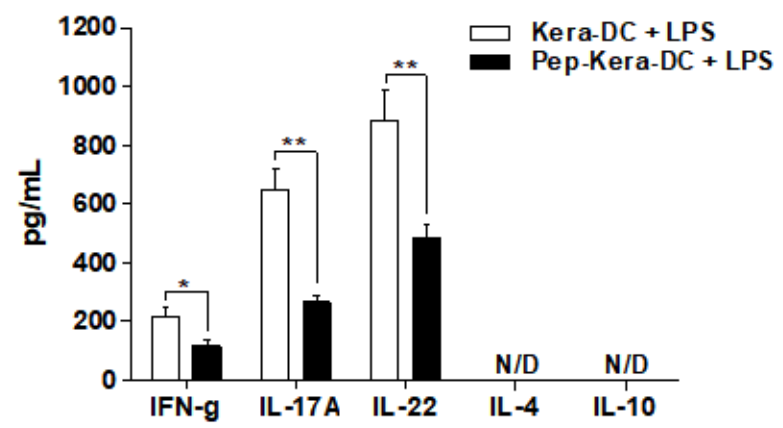

Figure 3.
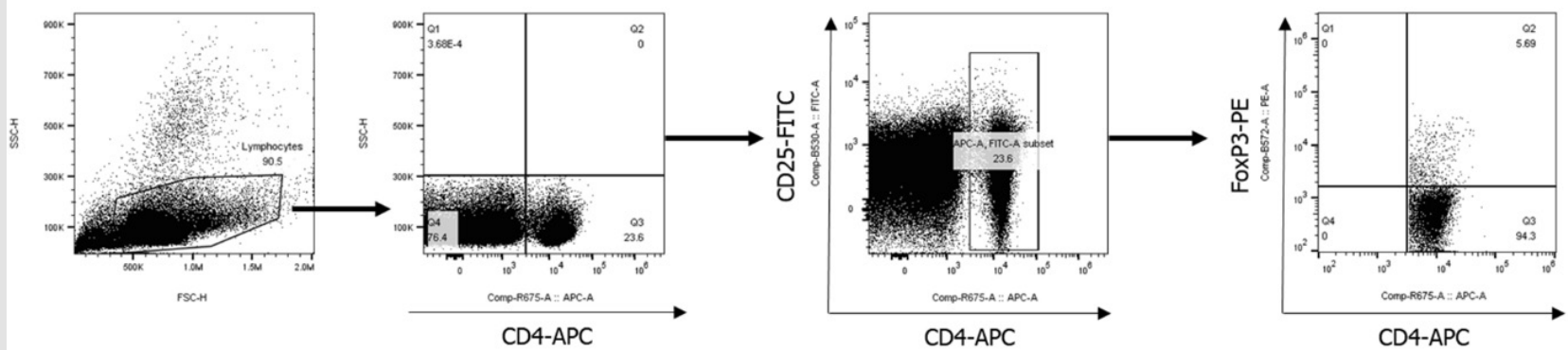

Figure 2S: Full gating strategy for flow cytometry data showing analysis of $\mathrm{CD} 4^{+} \mathrm{CD} 25^{+} \mathrm{Foxp} 3^{+}$cells gated on all lymphocytes. Initially, a broad scatter gate that excludes debris was applied. T cells were subdivided by gating on CD4 $4^{+}$populations. Foxp3 expression was quantified by gating positive populations.

Cell Culture Supernatant from AdhPep-10-2-Treated Keratinocytes Induces Anti-Inflammatory Responses by Blocking Activation of Effector T Cells

To investigate the effect of soluble factors produced by keratinocytes, we cultured splenocytes in the presence of keratinocyte culture supernatant. Supernatants from AdhPep10-2-treated keratinocytes (PKS) and from keratinocytes alone (KS) were concentrated 20-fold. Half of the splenocyte culture medium was replaced with each supernatant. Compared with KSsplenocytes, PKS-splenocytes reduced the population of $\mathrm{CD} 4^{+}$cells, $\mathrm{CD}^{+}$cells, and IFN- $\gamma$ secreting T cells (Figure 4A). PKS-splenocytes tended to induce Th2 cytokines (IL-4 and IL-10) rather than Th1 (IFN- $\gamma$ ) and Th17 (IL-17) cytokines (Figure 4B). The B cell and NK cell populations (Fig 4C) were not affected by the peptide. A full gating strategy is shown in Figure S3. Figures $3 \& 4$ imply that the PKS-splenocytes efficiently suppressed differentiation of effector $\mathrm{T}$ (Teff) cells.

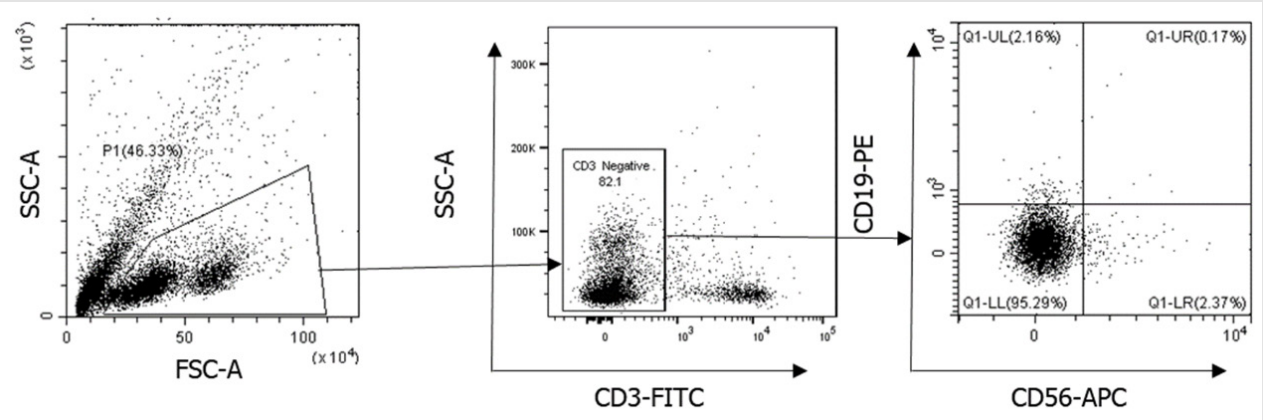

Figure 3S: Flow cytometry gating strategy for B cells and NK cells. Initially, a broad scatter gate that excludes debris was applied. T cells were excluded by CD3 expression. B cells and NK cells were then subdivided by gating on CD19 ${ }^{+}$and $\mathrm{CD} 56^{+}$ populations. 
(A)
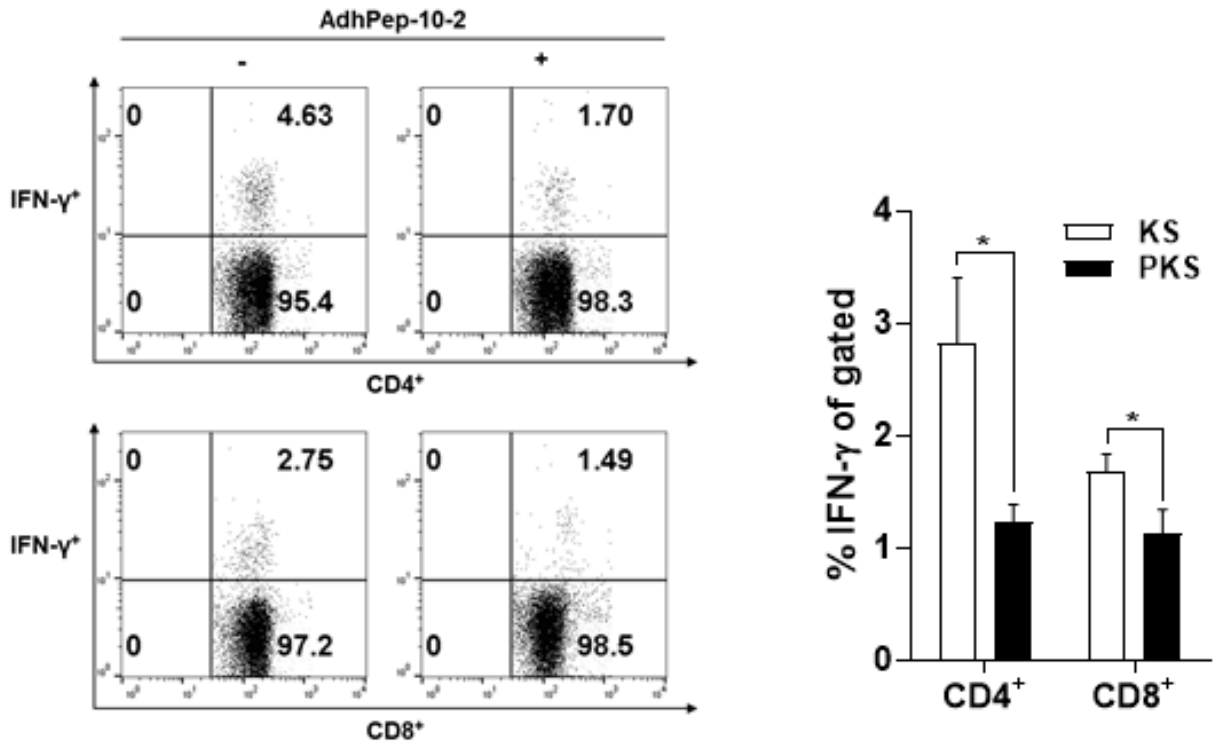

(B)

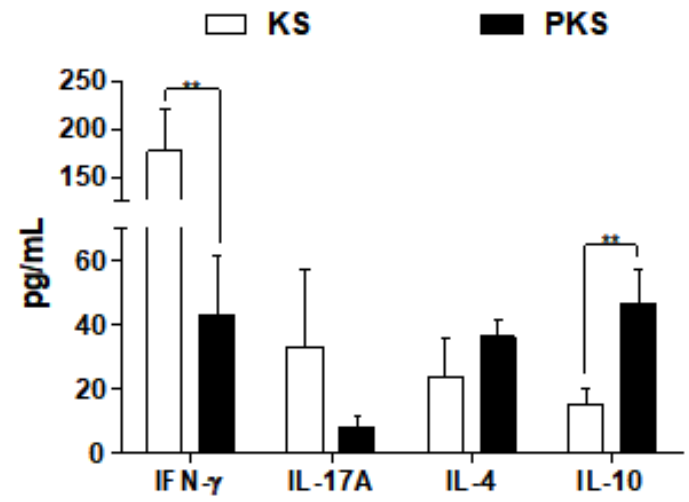

(C)
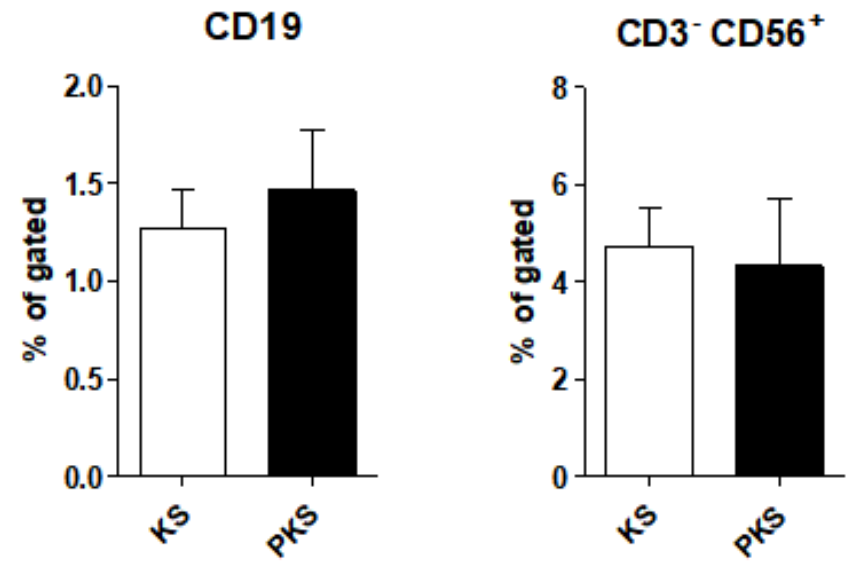

Figure 4. 
Immunoreactivity of Splenocytes Treated with Keratinocyte Culture Supernatant:

a) Splenocytes were treated for $24 \mathrm{~h}$ with keratinocyte culture supernatants, and the $\mathrm{CD} 4^{+} \mathrm{IFN}-\gamma^{+} \mathrm{T}$ cell or CD8 ${ }^{+} \mathrm{IFN}-\gamma^{+} \mathrm{T}$ cell populations were analyzed by flow cytometry (data are representative of three independent co-culture preparations). The bar graph shows the mean percentage of each cell type. Data are expressed as mean \pm SEM $(n=3$ co-culture preparations).

b) IFN- - , IL-17A, IL-4, and IL-10 in the culture supernatants of splenocytes, as measured by ELISA. Data are expressed as mean \pm SEM ( $n=3$ co-culture preparations).

c) $\mathrm{CD}^{+} 9^{+}$(B cells) and $\mathrm{CD}^{+} 6^{+}(\mathrm{NK}$ cells $)$ populations were analyzed by flow cytometry. The bar graph shows the mean percentage of each cell type. Data are expressed as mean \pm SEM ( $\mathrm{n}=3$ co-culture preparations). ${ }^{*} \mathrm{P}<0.05$; ${ }^{* *} \mathrm{P}<0.01$.

\section{AdhPep-10-2-Treated Keratinocytes Downregulate Production of CXCL1}

To identify why AdhPep-10-2-treated keratinocytes induce antiinflammatory response, we next performed a Luminex Multiplexing assay. There was no significant difference in production of proinflammatory cytokines between keratinocytes with AdhPep-10-2treated keratinocytes. However, AdhPep-10-2-treated keratinocytes effectively reduced production of CXCL1 without affecting that of pro-inflammatory cytokines (Figure 5A). Moreover, as measured by ELISA, AdhPep-10-2-treated keratinocytes produced significantly less CXCL1 (Figure 5B left) and CXCL1 mRNA levels were decreased in cell lysate by RT-PCR (Figure $5 \mathrm{~B}$ right). These results confirm that AdhPep-10-2-treated keratinocytes reduced production of CXCL1.

(A)

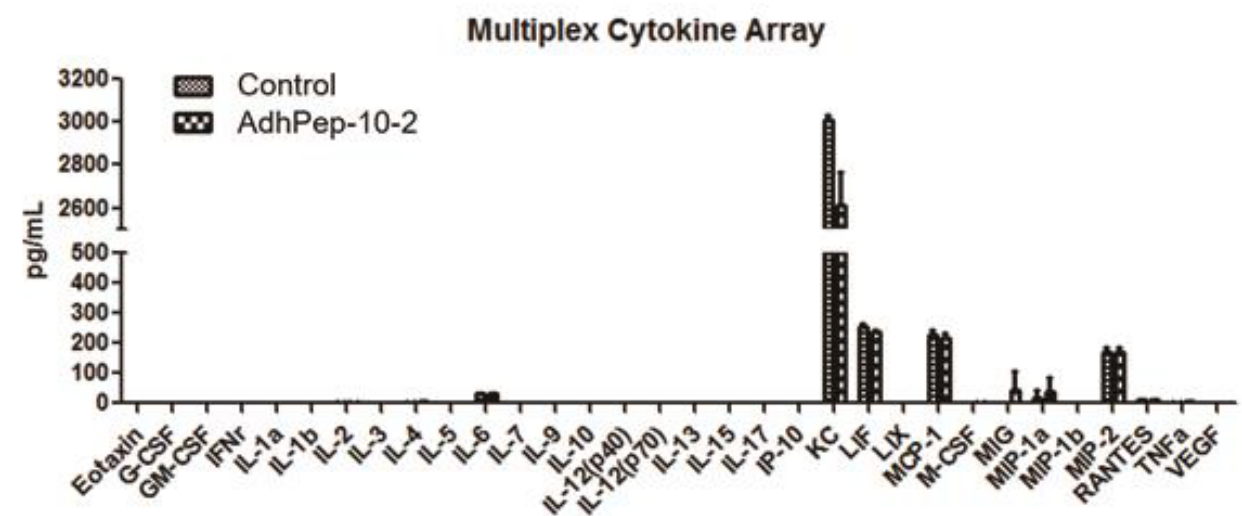

(B)
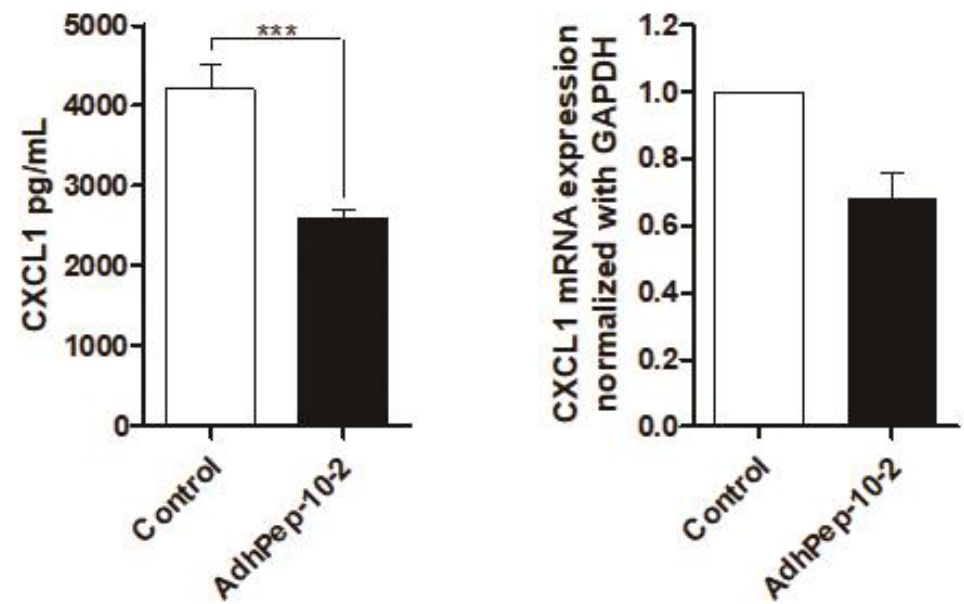

Figure 5. 
AdhPep-10-2-Treated Keratinocytes Reduced Production of CXCL1:

a) Culture supernatants from keratinocytes treated (or not) with AdhPep-10-2 were analyzed using a multiplex cytokine array (mean \pm SEM; $\mathrm{n}=3$ independent keratinocyte preparations).

b) CXCL1 levels in the supernatants of keratinocytes treated (or not) with AdhPep-10-2 were measured by ELISA (left panel) and CXCL1 mRNA levels in cell lysate were measured by RTPCR (right panel). Data are expressed as mean \pm SEM $(n=3$ independent co-culture preparations performed in duplicate) $* * * P<0.001$.

\section{Discussion}

Skin is a major entry site for microbial pathogens and allergens; therefore, it is heavily protected by DCs $[13,14]$. TJ proteins expressed by keratinocytes play a central role in barrier function. This affects not only epidermal barrier function and regulation by the immune system but is also relevant to skin diseases. The peptides, AdhPep-10-2, for cell conjugation efficiently promotes interaction between keratinocytes and increases expression of TJ proteins. Here, we examined the effect of AdhPep-10-2treated keratinocytes on DCs-mediated immunity. We found that AdhPep-10-2-treated keratinocytes induce anti-inflammatory response by blocking DC maturation and cytokine production. We also found that in the presence of LPS, Pep-Kera-DCs increased the FoxP $3{ }^{+} \mathrm{CD}^{+} \mathrm{CD} 25^{+}$Treg population and reduced the IFN $-\gamma^{+}$ producing T cell population. Therefore, the data suggest that PepKera-DCs induce anti-inflammatory response under conditions of inflammation.

Interestingly, we found that AdhPep-10-2-treated keratinocytes effectively reduced secretion of CXCL1 without affecting that of other pro-inflammatory cytokines. A previous report shows that in the epidermis, CXCL1 plays an important role in recruiting inflammatory cells during wound healing $[15,16]$. Therefore, we expect that CXCL1 is an important factor for DC-mediated immunity. Moreover, even though CXCL1 is present in the AdhPep10-2-treated keratinocyte supernatant at levels lower than that in non-treated keratinocyte supernatant, it is not the only component that affects DC maturation and function (Figure 5). Thus, it is likely that multiple factors act together to inhibit DC maturation. Since DCs express CXCR2, the receptor for CXCL1, CXCL1 could have a direct effect on DC maturation. Moreover, the signaling pathways activated by CXCL1 binding to CXCR2 on DCs are unknown $[15,17]$. Therefore, future studies will examine different factors and their effects of signaling pathways.

Skin DCs extend dendrites between keratinocytes to develop a dense network [18]. Activated skin DCs gain access to antigen by extending their dendrites through epidermal TJs [19]. Keratinocyte TJs can be penetrated by these dendrites $[8,18]$. AdhPep10-2 increases expression of TJ proteins. We expect that in the skin, AdhPep-10-2 increases expression of TJ proteins by keratinocytes, thereby suppressing penetration by skin DC dendrites and lymphocytes. This has important implications for the pathogenesis of inflammatory skin diseases such as psoriasis and atopic dermatitis and for the healing of skin lesions. In fact, increased expression of CXCL1 has been reported in inflammatory skin diseases such as psoriasis [15,20,21]; indeed, it is suggested to play a role in neutrophil recruitment and epidermal hyperproliferation, which are characteristic of this disease. Furthermore, the number of CD $11 \mathrm{c}^{+}$DCs in the dermis of psoriatic lesions is 30fold higher than that in healthy skin $[1,15,20]$. Therefore, there are several potential avenues for a therapeutic intervention targeting augmented expression of CXCL1 and lymphocyte recruitment.

\section{Conclusion}

we showed here that AdhPep-10-2-treated keratinocytes inhibit DC maturation and increase the Treg cell population. These results show the possibility that increased keratinocyte TJ proteins inhibit DC maturation and inflammatory mediator production. Although the underlying mechanisms still need to be elucidated, we suggest the involvement of CXCL1 which expression is reduced.

\section{Author Contributions}

Conceptualization: Jun-Ho Lee, So-Yeon Choi, Soo-Yeoun Park, Dae-Seog Lim, Ji-Hyung Chung

Data curation: Nam-Chul Jung, Han Geuk Seo, Dae-Seog Lim

Investigation: Jun-Ho Lee, So-Yeon Choi, Soo-Yeoun Park, Ji-Soo $\mathrm{Oh}$

Project Administration: Jun-Ho Lee, So-Yeon Choi, Dae-Seog $\operatorname{Lim}$

Visualization: Soo-Yeoun Park, Jinjung Choi, Jie-Young Song

Writing: original draft: Jun-Ho Lee, So-Yeon Choi, Dae-Seog Lim

Writing: review \& editing: Jun-Ho Lee, So-Yeon Choi, Dae-Seog Lim, Ji-Soo Oh.

\section{Acknowledgment}

This research was supported by a grant of Korea Health Technology R\&D Project through the Korea Health Industry Development Institute (KHIDI), funded by Ministry of Health \& Welfare, Republic of Korea (grant number: HN14C0082).

\section{Conflict of Interest}

The authors declare no potential conflict of interest. 


\section{References}

1. Bangert C, Brunner PM, Stingl G (2011) Immune functions of the skin. Clin Dermatol 29(4): 360-376.

2. Pasparakis M, Haase I, Nestle FO (2014) Mechanisms regulating skin immunity and inflammation. Nat Rev Immunol 14(5): 289-301.

3. Sugita K, Kabashima K, Atarashi K, Shimauchi T, Kobayashi M, et al (2007) Innate immunity mediated by epidermal keratinocytes promotes acquired immunity involving Langerhans cells and T cells in the skin. Clin Exp Immunol 147(1): 176-183.

4. Lim DS, Kang MS, Jeong JA, Bae YS (2009) Semi-mature DC are immunogenic and not tolerogenic when inoculated at a high dose in collagen-induced arthritis mice. Eur J Immunol 39(5): 1334-1343.

5. Byun SH, Lee JH, Jung NC, Choi HJ, Song JY, et al. (2016) Rosiglitazonemediated dendritic cells ameliorate collagen-induced arthritis in mice. Biochem Pharmacol 115: 85-93.

6. Lee JH, Kim TH, Park HE, Lee EG, Jung NC, et al. (2014) Myosin-primed tolerogenic dendritic cells ameliorate experimental autoimmune myocarditis. Cardiovasc Res 101(2): 203-210.

7. Chu CC, Ali N, Karagiannis P, Di Meglio P, Skowera A, et al. (2012) Resident CD141 (BDCA3)+ dendritic cells in human skin produce IL10 and induce regulatory $\mathrm{T}$ cells that suppress skin inflammation. J Exp Med 209(5): 935-945.

8. Kubo A, Nagao K, Yokouchi M, Sasaki H, Amagai M (2009) External antigen uptake by Langerhans cells with reorganization of epidermal tight junction barriers. J Exp Med 206(13): 2937-2946.

9. Ji Hyung Chung (2018) Inventor; CHA University Industry-Academic Cooperation Foundation and CHABIO F \& C Co., Ltd., assignee. Novel peptides and cosmetic compositions for inhibiting skin aging or wrinkle formation comprising the same. Republic of Korea patent KR 1018172440000

10. Ji Hyung Chung, Da Yoon Chung (2018) Inventor; CHA University Industry-Academic Cooperation Foundation and CHABIO F \&C Co., Ltd., assignee. Novel peptides and cosmetic compositions for inhibiting skin aging or wrinkle formation comprising the same. Republic of Korea patent KR 1018172500000.

\section{ISSN: 2574-1241}

\section{DOI: 10.26717/BJSTR.2021.36.005820}

Dae-Seog Lim. Biomed J Sci \& Tech Res

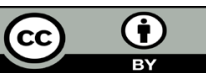

This work is licensed under Creative Commons Attribution 4.0 License

Submission Link: https://biomedres.us/submit-manuscript.php
11.Ji Hyung Chung (2019) Inventor; College of Medicine Pochon CHA University IndustryAcademic Cooperation Foundation, assignee. Peptide and cosmetic composition comprising same for preventing skin aging or skin wrinkle formation. United States patent US 10478392.

12. Chung, Ji Hyung (2016) Inventor; College of Medicine Pochon Cha University IndustryAcademic Cooperation Foundation, assignee. Novel Peptide and Cosmetic Composition Comprising Same for Preventing Skin Aging or Skin Wrinkle Formation. European Patent EP 16860242.3.

13. Malissen B, Tamoutounour S, Henri S (2014) The origins and functions of dendritic cells and macrophages in the skin. Nat Rev Immunol 14(6): 417-428.

14. Merad M, Ginhoux F, Collin M (2008) Origin, homeostasis and function of Langerhans cells and other langerin-expressing dendritic cells. Nat Rev Immunol 8(12): 935-947.

15. Faleiros RR, Leise BB, Westerman T, Yin C, Nuovo GJ, et al. (2009) In vivo and in vitro evidence of the involvement of CXCL1, a keratinocytederived chemokine, in equine laminitis. J Vet Intern Med 23(5): 10861096.

16. Gillitzer R, Goebeler M (2001) Chemokines in cutaneous wound healing. J Leukoc Biol 69(4): 513-521.

17. Dastmalchi F, Karachi A, Yang C, Azari H, Sayour EJ, et al. (2019) Sarcosine promotes trafficking of dendritic cells and improves efficacy of anti-tumor dendritic cell vaccines via CXC chemokine family signaling. J Immunother Cancer 7(1): 321.

18. Rescigno M, Urbano M, Valzasina B, Francolini M, Rotta G, et al. (2001) Dendritic cells express tight junction proteins and penetrate gut epithelial monolayers to sample bacteria. Nat Immunol 2(4): 361-367.

19. Hennen J, Aeby P, Goebel C, Schettgen T, Oberli A, et al. (2011) Cross talk between keratinocytes and dendritic cells: impact on the prediction of sensitization. Toxicol Sci 123(2): 501-510.

20. Lowes MA, Bowcock AM, Krueger JG (2007) Pathogenesis and therapy of psoriasis. Nature 445(7130): 866-873.

21. Homey B, Dieu Nosjean MC, Wiesenborn A, Massacrier C, Pin JJ, et al. (2000) Upregulation of macrophage inflammatory protein-3 alpha/ CCL20 and CC chemokine receptor 6 in psoriasis. J Immunol 164(12): 6621-6632.

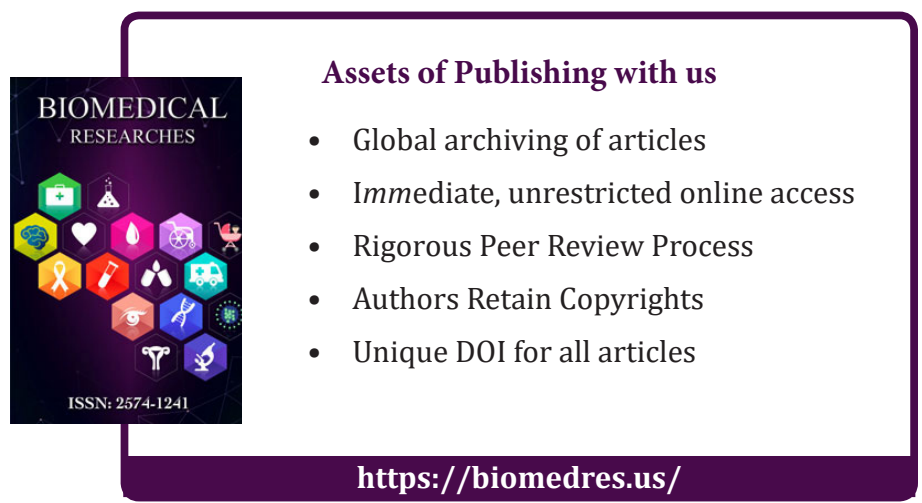

